\title{
A Data Collection Algorithm based on Balanced Energy Consumption Efficiency of Wireless Sensor Network
}

\author{
Zujie Bie* and Feng Pan
}

Department of information, Chongqing University of Science \& Technology, Chongqing 401331, P.R. China

\begin{abstract}
Wireless sensor network resources are limited, node energy consumption imbalance between sensor nodes, the overall network life cycle shorten. For wireless sensor network energy consumption imbalance of the problems in the process of data collection, proposed a kind of algorithm based on energy equilibrium and efficient data collection. The algorithm of the network deployment area is divided into size grid, and on the basis of the residual energy of nodes and the cluster head rotation, then uses the data fusion technology, can effectively improve the node energy consumption balance and prolong the network lifetime significantly. Simulation and performance analysis results show that compared with the typical data collection algorithms, the algorithm has a better performance in the aspect of balance of energy consumption and the network life cycle.
\end{abstract}

Keywords: Data collection, data fusion, energy consumption balanced, remaining energy, wireless sensor network.

\section{INTRODUCTION}

Wireless sensor network (Wireless Sensor Network, WSN) is a random distribution in the area of the monitoring of a large number of sensor nodes, using Wireless communication technology of self-organizing network system [1]. Through the sensor nodes common perception, collection and processing of in network coverage area information by monitoring object, enables people to get reliable under any circumstances surrounding environment information, is used widely in battlefield surveillance, medical monitoring, traffic monitoring and environmental monitoring, etc. But, due to the wireless sensor network node is smaller, there are drawbacks, such as communication, computing and storage capacity is limited and sensor nodes usually only equipped with energy limited battery, mostly deployed in no one guard in a more sinister dangerous remote environment, makes the regular change of sensor nodes do not have the feasibility. How to solve the problem of sensor node energy efficiency which is became the difficult point of wireless sensor network (WSN) research. In the process of data collection and transmission, data by cluster head near the sensor nodes to collect more data of ordinary sensor nodes, by removing redundant data fusion processing before forwarding, is a very effective energy saving techniques [2-4]. However, because of the cluster head nodes for more tasks such as computing, storage, communications, energy consumption than ordinary nodes faster, caused network energy consumption uneven, premature deaths in the network of nodes, data collection may arose the black hole, so as to speed up the shorter working life of the wireless sensor network as a whole. Therefore, the network node energy consumption too fast and the network energy consumption imbalance become hotspot and difficult problem of wireless sensor network (WSN) research.

\section{RELATED RESEARCH}

Data fusion technology is a kind of energy-saving technology base on data collection and transmission, however, because of the need for integration operation of cluster head sensor node energy consumption too fast, in the network of nodes premature end of life, thus improving for the overall network performance and life time is not very big. Especially after the new cluster head election in some more rounds, with the increase of node number of deaths, network performance more decline substantially. Thus, the cluster head rotation technology is a way to balance energy consumption in data collection, can effectively prolong network life time. At present, according to the techniques used in different ways, the method of cluster head rotation can be divided into three types:

Periodic press wheel random cluster head election rotation cluster heads, take the energy of the cluster head load distribution on each ordinary sensor nodes. Main typical representative of the agreement such as LEACH (Low Energy Adaptive Clustering Hierarchy) [5] agreement, through the new cluster head nodes are randomly selected from the network, the common nodes choose the nearest cluster head to join the cluster become the members of cluster, cluster head rotation for the next round of new variety selection and preparation for construction work. However, this type of scheme does not consider the network node residual energy problems, if some smaller residual energy of nodes is selected as the cluster head in the rotation, will speed up the node death, thus reducing the overall network performance.

Through the building homogeneous clusters of similar size to cluster head rotation, let each cluster head nodes in the receiving and sending data of clusters of energy con- 
sumption similar. Main typical representative of the agreement has HEED (Hybrid Energy Efficient Distributed clustering) [6] agreement, on the basis of the residual energy of nodes to select cluster head, and then according to the proximity of the node to the neighbor node and the node degrees of building cluster of similar size. However, this type of scheme does not consider the distance different caused energy consumption imbalance problem between each cluster to the base station, distance far bunch of cluster head nodes and the base station distance from sending data and to consume more energy and will form "hotspots" [7], cause far away earlier clusters will not work.

Through building sizes of non-uniform cluster to cluster head rotation, considering the distance and the energy consumption of sending and receiving data for each cluster of cluster head energy equilibrium. Main typical representative of the agreement has DEEUC (Distributed Energy Efficient Unequal Clustering) [8] agreement, with the introduction of balance factor to improve the more residual energy of nodes become the probability of candidate cluster head, by each candidate cluster head nodes with the same radius of broadcast news competition of cluster head again, if the other candidate received the residual energy of cluster head more candidate cluster head message sent by the competition, then the candidate cluster head automatic give up competition become common node, the remainder of the competition after the candidate cluster head to become a cluster head, according to the common node and the distance of the cluster head and base station to choose the appropriate cluster to join the cluster head. However, this type of scheme is mainly consider the balance of power between cluster head, do not consider common node selection cluster of energy consumption caused by the distance too far too fast earlier death, ordinary nodes is not always choose the nearest cluster head and join the cluster, if it chooses distance too far for cluster head, will consume more energy than the other cluster members to send data, will reduce the efficiency of energy use.

\section{THE NETWORK MODEL}

Assume that wireless sensor network is composed of $m$ sensor node, will randomly deployed evenly in a plane coordinate system of long for $a$, wide for $b$ and starting point for $\left(O_{x}, O_{y}\right)$ of the rectangular area, and has the following properties:

All sensor nodes time synchronization, the position unchanged after deployment;

All sensor nodes are homogeneous and have unique identifiers;

All sensor nodes have the same energy and computing power handling and routing calculation;

All sensor nodes can adjust itself transmission power according to the communication distance;

The base station and all the sensor nodes can accord the received signal strength to learn the coordinates of your location;
The base station is located outer the node deployment area, coordinates for $\left(B_{x}, B_{y}\right)$, and is also the position unchanged after the deployment;

Using energy consumption model in literature [9], calculate the sensor node sends data energy consumption $E_{T_{x}}$ and receiving data energy consumption $E_{R x}$ and fusion data energy consumption $E_{A}$. Such as formula (1), formula (2) and formula (3).

$$
\begin{aligned}
& E_{T x}(n, s)=\left\{\begin{array}{l}
n \times E_{\text {elec }}+n \times \lambda_{f s} \times s^{2}, s<s_{0} \\
n \times E_{\text {elec }}+n \times \lambda_{m p} \times s^{4}, s \geq s_{0}
\end{array}\right. \\
& E_{R x}(n)=n \times E_{\text {elec }} \\
& E_{A}(q, n)=q \times n \times E_{D A}
\end{aligned}
$$

Among them, $n$ shows transmit data bit length, $s$ shows transmission distance, $s_{0}$ shows default transmission distance threshold, $E_{\text {elec }}$ shows transmission unit length data needed for energy consumption, $\lambda_{f s}$ shows under the free space model condition of the energy consumption parameters, $\lambda_{m p}$ shows the energy consumption parameters under the condition of multipath fading model, $q$ shows the number of data required to fusion, $E_{D A}$ shows the fusion of unit length data needed for energy consumption.

\section{THE DATA COLLECTION ALGORITHM DESIGN OF THE BALANCED ENERGY CONSUMPTION}

Wireless sensor network (WSN) may occur in the operation of the work in the life cycle energy consumption imbalance of reason mainly has the following four aspects:

Because of sending distance different causes energy consumption imbalance between cluster members;

Between cluster head and cluster members causes energy consumption imbalance by different operating; between cluster head causes energy consumption imbalance by receiving data different;

Between cluster head causes energy consumption imbalance by data sending distance different.

At present in the data collection, data fusion technology adopted the general cluster head rotation algorithm only can try to meet the demand of the energy consumption in a certain respect of the balance. Starting from the various balanced energy consumption target of overall network, gives a kind of efficient energy consumption equilibrium data collection algorithms (Data Gathering algorithm based on Balanced and Efficiency Energy Dissipation, DGBEED), according to the energy consumption characteristics of different regional cluster head, adjust to participate in each round the node number of the cluster head rotation. Through the wireless sensor network is divided into different sizes of non-equal size of area, the area of the farther from the base station, the greater the area divided into, contains the more involved in the selection of cluster head nodes, then each node residual energy according to the area choose one of the 
biggest residual energy node as cluster-heads, ordinary node can choose the nearest cluster head to join the cluster. The DGBEED algorithm is given below description, as shown in Algorithm 1.

The algorithm 1: DGBEED algorithm description.

Network distribution areas division

1.1) First of all, along toward the direction of the base station divided the network into $M$ equal strip area, each strip area with $M$ consecutive integers are identified;

1.2) Second, along toward the vertical direction of the base station area for $M$ strips according to the distance with the base station divided into different sizes of lattice area, the farther from the base station of the strip area, divided is less by the lattice area, the greater the area of the grating region;

1.3) Finally, from the base station to all sensor nodes broadcast network areas classification news and node location information according to the message and its own computing own lattice area information, each lattice area all nodes to form a cluster.

\section{2) Cluster head selection and rotation}

2.1) The first round of cluster head collaboration select by each lattice area nodes, all nodes within the cluster to cluster in a fixed range of broadcasting information about themselves, including nodes position information, the node itself belongs to cluster and residual energy information, each node receives only belong to this cluster node information, then you can select this cluster of cluster head;

2.2) The non-first round of cluster head elected by round of cluster head on collaboration, each round the last data collection, each cluster member take their remaining energy information such as "piggyback" sent to the cluster head, the previous round of cluster head nodes according to their cluster classification, and then select candidate cluster head for each cluster, and round up the cluster head send candidate cluster head nodes information, this cluster of the previous round of cluster head select this cluster of new cluster head;

2.3) After the completion of the new round of cluster head, the new cluster head toward the scope of the cluster's radio himself as a variety of information, the common nodes according to receive the new cluster head of broadcasting information to choose a nearest cluster head and join the cluster, and then the new cluster head send the data transmission time slot for this cluster members;

\section{3) Collection of data fusion}

3.1) After each round of the new cluster head election, network tend to be stability, each cluster member within its own transmission time slot will collect data sent to the cluster head, are sent to the base station after fusion of cluster head treatment, before the next round of cluster head rotation can be many times the same data collection;

3.2) The last data collection of each round, all the cluster members attach their remaining energy information in the collection of data information, "piggyback" sent to the cluster head, as the next round of cluster head rotation reference information.

\section{THE SIMULATION AND PERFORMANCE ANALYSIS}

The DGBEED algorithm in this paper, we divided the wireless sensor network into different sizes of lattice area, the farther from the base station, the greater the area of the grating region, contains more nodes to participate in the cluster head rotation, so as to share the energy consumption load of the cluster head, also can reduce the distance between the base stations far away the energy of the cluster head send the data, as long as there is no energy depletion of nodes can according to the size of the residual energy of the remaining nodes to select a new cluster head, so not only ensure the regularity of each rotation number of cluster head, but also ensure the distribution regularity of cluster head in each rotation. In addition to, using "piggyback" and limit node transmission distance way also can save the sensor node energy effectively. Below by adopting OPNET10.0 simulation comparison with DGBEED algorithm and DEEUC algorithm as the number of cluster head rotation amount of remaining energy increasing network and live node number change. Among them, the parameters of the initial value for: network coverage area is $300 \mathrm{~m} \times 200 \mathrm{~m}$, the node number is 500 , the initial energy of each node is $0.4 \mathrm{~J}$.

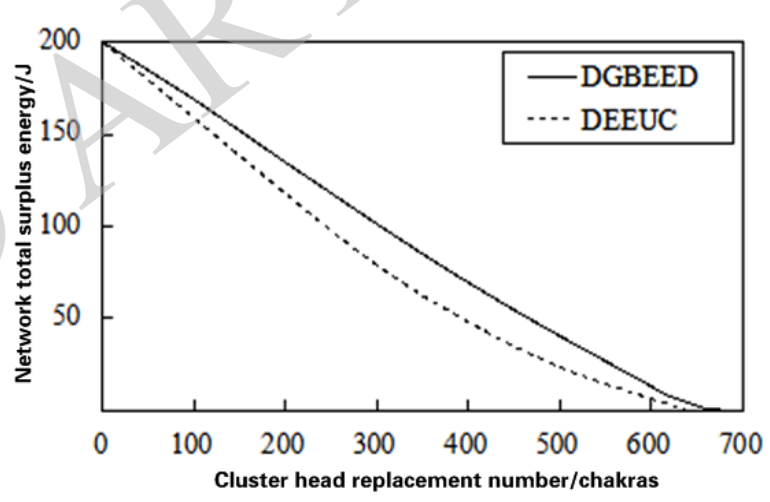

Fig. (1). Increase total change of network surplus energy with round number change.

Can be seen from Fig. (1), with each round of the new cluster head replacement, DGBEED algorithm and DEEUC algorithm under the overall network are gradually reduce the total surplus energy, and energy consumption of DGBEED algorithm is relatively flat, DEEUC algorithm energy consumption with replacement round by the increase in the number dropped sharply, a sharp fall in the number of diagrammatic curve. Under the same number of cluster head replacement round, DGBEED algorithm of the residual total energy is higher than the DEEUC algorithm. And in the case of DEEUC algorithm of total surplus energy than earlier depleted DGBEED algorithm, thus the wireless sensor network using DGBEED algorithm will be able to get a more lasting life survival time can be seen from the Fig. (2), each sensor node energy in early stage can work for a period of time, during this period showed little change network work node number of survival. With each round of the new cluster head replacement, however, because each node energy consumption is different, especially the cluster head nodes take more energy consumption, network after work a period of time, DGBEED algorithm and DEEUC algorithm under the live network node number are in decline, but in the same 
round number, the DGBEED algorithm presented node number of survival is higher than DEEUC algorithm. And the DEEUC algorithm of node premature death and survival number reduced quickly, and DGBEED algorithm of node death late and periodically drop, so wireless sensor network (WSN)'s performance is better than the DEEUC algorithm under the DGBEED algorithm. At the same time, also can see all the network nodes of the DEEUC algorithm earlier will not work, network will be able to get more lasting working life time under the DGBEED algorithm.

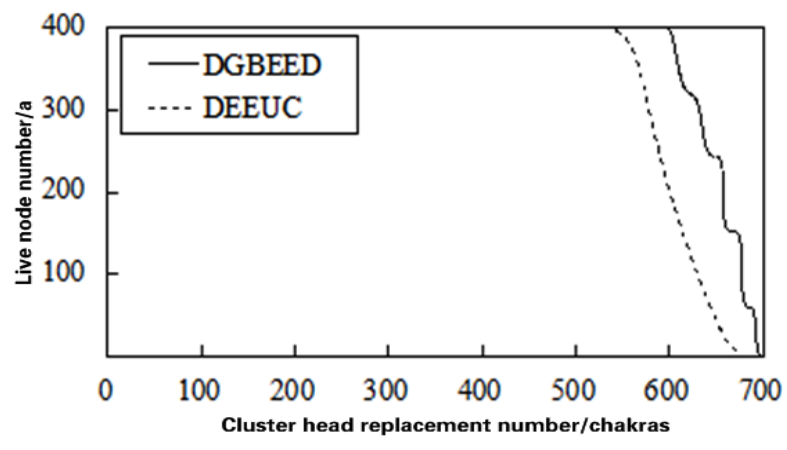

Fig. (2). Increase number change of network survival node with round number.

\section{CONCLUSION}

Data fusion is an effective energy-saving technology in wireless sensor network (WSN), but the collected data is mainly consisted of cluster head by the data fusion, which creates a cluster head nodes energy consumption speed up the possible early failure. It may result in the network performance decrease of energy consumption and cause unbalanced and short network life cycle. We propose the DGBEED algorithm in this paper. Through the lattice area of network partitioning size, the farther the distance between the base station is, the larger the lattice area becomes. The rotation of cluster head nodes can be a good balance between the cluster energy consumption. Energy consumption between the members of cluster, the cluster head, the network performance, network energy use efficiency and life span can make the network achieve longer balanced. The energy consumption target has a very good practical application value.

\section{CONFLICT OF INTEREST}

The authors confirm that this article content has no conflict of interest.

\section{ACKNOWLEDGEMENTS}

This work is supported by Chongqing natural science fund project (cstc2012jjA40025).

\section{REFERENCES}

[1] J. Kan, X.Z. Zuo, and L.W. Tan, "Wireless sensor network data fusion technology," Computer Science, vol. 37, no. 4, pp. 31-35, 2010.

[2] S.D. Zhan, and P.W. Huang, "Facing the network survival time delayed allocation algorithm of sensor network integration," Research and Development of the Computer, vol. 47(Suppl), pp. 224228, 2010.

[3] Z.Y. Chen, G. Yan, and L. Chen, "Wireless sensor network data fusion research reviewed," Computer Application Research, vol. 28, no. 5, pp. 1601-1613, 2011.

[4] Y.G. Yi, and G.G. Din, "Research on data compression technology in wireless sensor network," Computer Applications and Software, vol. 27, no. 7 , pp. 1-4, 2010.

[5] W. Heinzelman, A. Chandrakasan, and H. Balakrishnan, "Energyefficient communication protocol for wireless micro-sensor networks," In: Proceedings of the Conference on System Sciences, 2000, pp. 3005-3014.

[6] O. Younis, and S. Fahmy, "Heed: a hybrid, energy-efficient, distributed clustering approach for ad hoc sensor networks," IEEE Transaction on Mobile Computing, vol. 3, no. 4, pp. 366-379, 2004.

[7] S. Soro, and W. Heinzelman, "Prolonging the lifetime of wireless sensor networks via unequal clustering," In: Proceedings of the Parallel and Distributed Processing Symposium, 2005, pp. 1-8.

[8] F.J. Shang, M. Abolhasan, and T. Wysocki, "Wireless sensor network (WSN) are distributed energy efficient heterogeneous clusters algorithm," Journal of Communication, vol. 30, no. 10, pp. 34-43, 2009.

[9] Z.X. Liu, Q.C. Zheng, and L. Xue, Energy and node degree synthesized clustering algorithm for wireless sensor networks," Journal of Software, vol. 20 (Suppl), pp. 250-256, 2009.

Received: June 10, 2015

Revised: July 29, 2015

Accepted: August 15, 2015

C Bie and Pan; Licensee Bentham Open.

This is an open access article licensed under the terms of the Creative Commons Attribution Non-Commercial License (http://creativecommons.org/licenses/by-nc/3.0/) which permits unrestricted, non-commercial use, distribution and reproduction in any medium, provided the work is properly cited. 